\title{
Cholesterol favors phase separation of sphingomyelin
}

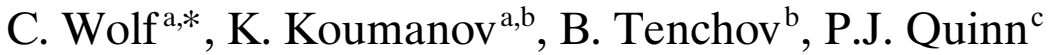 \\ ${ }^{a}$ Biochimie, INSERM U538, Laboratoire Commun de Spectrometrie, Faculté de Médecine de Saint Antoine, 27 rue \\ Chaligny, Paris, 75012 France \\ ${ }^{\mathrm{b}}$ Institute of Biophysics, Bulgarian Academy of Sciences, Sofia, 1113 Bulgaria \\ ${ }^{\mathrm{c}}$ Biochemistry Department, King's College, London, UK
}

Received 14 August 2000; received in revised form 17 October 2000; accepted 20 October 2000

\begin{abstract}
The phase behavior of mixed lipid dispersions representing the inner leaflet of the cell membrane has been characterized by X-ray diffraction. Aqueous dispersions of phosphatidylethanolamine:phosphatidylserine (4:1 mole/mole) have a heterogeneous structure comprising an inverted hexagonal phase $\mathrm{H}_{\mathrm{II}}$ and a lamellar phase. Both phases coexist in the temperature range $20-45^{\circ} \mathrm{C}$. The fluid-to-gel mid-transition temperature of the lamellar phase assigned to phosphatidylserine is decreased from 27 to $24^{\circ} \mathrm{C}$ in the presence of calcium. Addition of sphingomyelin to phosphatidylethanolamine/phosphatidylserine prevents phase separation of the hexagonal $\mathrm{H}_{\text {II }}$ phase of phosphatidylethanolamine but the ternary mixture phase separates into two lamellar phases of periodcity 6.2 and $5.6 \mathrm{~nm}$, respectively. The 6.2-nm periodicity is assigned to the gel phase enriched in sphingomyelin of molecular species comprising predominantly long saturated hydrocarbon chains because it undergoes a gel-to-fluid phase transition above $40^{\circ} \mathrm{C}$. The coexisting fluid phase we assign to phosphatidylethanolamine and phosphatidylserine and low melting point molecular species of sphingomyelin which suppresses the tendency of phosphatidylethanolamine to phase-separate into hexagonal $\mathrm{H}_{\mathrm{II}}$ structure. There is evidence for considerable hysteresis in the separation of lamellar fluid and gel phases during cooling. The addition of cholesterol prevents phase separation of the gel phase of high melting point sphingomyelin in mixtures with phosphatidylserine and phosphatidylethanolamine. In the quaternary mixture the lamellar fluid phase, however, is phase separated into two lamellar phases of periodicities of 6.3 and $5.6 \mathrm{~nm}\left(20^{\circ} \mathrm{C}\right)$, respectively. The lamellar phase of periodicity $5.6 \mathrm{~nm}$ is assigned to a phase enriched in aminoglycerophospholipids and the periodicity $6.3 \mathrm{~nm}$ to a liquid-ordered phase formed from cholesterol and high
\end{abstract}

Abbreviations: SAXS, small angle X-ray scattering; WAXS, wide angle X-ray scattering; CHOL, cholesterol; SM, sphingomyelin; PE, phosphatidylethanolamine; PS, phosphatidylserine; 7DHC, 7-dehydrocholesterol.

* Corresponding author. Tel.: + 33-1-4001-1490; fax: +33-1-4001-1499.

E-mail address: wolf@ccr.jussieu.fr (C. Wolf). 
melting point molecular species of sphingomyelin characterized previously by ESR. Substituting 7-dehydrocholesterol for cholesterol did not result in evidence for lamellar phase separation in the mixture within the temperature range $20-40^{\circ} \mathrm{C}$. The specificity of cholesterol in creation of liquid-ordered lamellar phase is inferred. (C) 2001 Elsevier Science B.V. All rights reserved.

Keywords: X-Ray diffraction; Lipid phase; Microdomains; 7-Dehydrocholesterol; Smith Lemli Opitz syndrome

\section{Introduction}

The presence of cholesterol (CHOL) in cell membranes was initially thought to act as a homogenizing agent of the membrane matrix [1] which is comprised of a remarkable variety of molecular species of phospholipids, each with a distinct gel/fluid transition temperature. The alteration of membrane lipid fluidity brought about by cholesterol is assumed to minimize the difference between fluid and gel states of the phospholipid acyl chains, which may be expected to favour the mixing of membrane lipids at intermediate temperatures. A different role for $\mathrm{CHOL}$ is now envisaged in which the sterol interacts specifically with high melting temperature sphingolipids and promotes their phase separation into domains of liquid-ordered lamellar phase $\left(L_{\mathrm{o}}\right)$. These domains represent the immiscible rafts of the lipid matrix of biological membranes [2-6]. The stabilization by CHOL of the $L_{\mathrm{o}}$ phase has been shown to correlate with the resistance of the structures to disruption by non-ionic detergents at low temperatures during their isolation from biological membranes [7].

The interaction of CHOL involves multiple van der Waals contacts of the polycyclic ring with the extended saturated acyl chains of molecular species of sphingolipids which form a gel phase up to physiological temperatures [8]. The specificity of the interaction with regard to the sterol structure has been recently described and two distinct categories have been proposed: (i) sterols like cholesterol which promote phase-separation of $L_{\mathrm{o}}$ phase; and (ii) other sterol structures that inhibit the packing with saturated lipids and act as 'anti-cholesterol' [9].

The present study was undertaken to determine whether partitioning of SM and CHOL may be modulated by alternative interactions with glycerophospholipids. The aim was to model as closely as possible the highly complex lipid matrix of biological membranes which are comprised of hundreds of molecular species and different lipid classes. Detailed studies have already been reported of the phase behavior of model lipids, synthetic or purified, as a single molecular species or as relatively simple codispersion [10,11]. These have indicated that $\mathrm{CHOL}$ is readily miscible within lecithin and sphingomyelin bilayers simple codispersions [10] whereas solubility in phosphatidylethanolamine or phosphatidylserine is limited [12-15]. This suggests that the phase equilibrium in mixtures comprising SM, CHOL and aminophospholipids is a more realistic model of the cytoplasmic leaflet of the plasma membrane than simple binary mixtures [16].

Using synchrotron X-ray diffraction methods we have been able to detect the phase-separation of liquid-ordered SM/CHOL lamellae within mixed fluid amino-glycerophospholipids. Aminoglycerophospholipids (phosphatidylethanolamine (PE) and phosphatidylserine (PS)) in an aqueous dispersion are known to undergo lamellar/nonlamellar $\left(\mathrm{H}_{\mathrm{II}}\right)$ phase transitions at characteristic temperatures influenced by calcium and cholesterol $[17,18]$.

The association of CHOL with SM and consequent modifications of phase-equilibrium in the heterogeneous mixture $\mathrm{PE} / \mathrm{PS} / \mathrm{SM}$ have been characterized during temperature scans by synchrotron X-ray diffraction providing information on the long-range order resolved in the phaseseparated lipid mixtures. Temperature scans are used to monitor the thermotropic phase-separation around physiological temperatures and to detect hysteresis in phase behavior. The results indicate specific interactions take place between CHOL and SM but not between SM and 7-dehy- 
drocholesterol, in which no phase separation was observed.

\section{Materials and methods}

Lipids (egg-yolk phosphatidylethanolamine, egg sphingomyelin, brain phosphatidylserine and sterols) were purchased from Sigma (L'Isle d'Abeau, 38297 Saint-Quentin Fallavier, France). The fatty acid composition of egg SM determined by gas chromatography-mass spectrometry as described by Katsikas and Wolf [19] is: C16:0, 78\%; C18:0, 7\%; C20:0, 2\%; C22:0, 4\%; C24:0, 2\%; $\mathrm{C} 24: 1,3 \%$.

The dry lipids were dissolved in $\mathrm{CHCl}_{3}$ and mixed in the desired proportions indicated throughout the text as mole ratio. The solvent was evaporated under a stream of oxygen-free dry nitrogen and the remaining traces of solvent removed by storage at $20^{\circ} \mathrm{C}$ in vacuo. The dry lipid mixtures were hydrated with $100 \%(\mathrm{w} / \mathrm{w})$ of the aqueous buffer $100 \mathrm{mM}$ Tris- $\mathrm{HCl}, \mathrm{pH} 8$, containing $10 \mathrm{mM} \mathrm{CaCl} 2$. The dispersions were repeatedly frozen and thawed before being stored for several days at room temperature then at $4^{\circ} \mathrm{C}$ under argon before examination to prevent an artifact due to an incomplete mixing and/or hydration of the samples.

Synchrotron X-ray diffraction measurements were performed at station 8.2 of the Daresbury Synchrotron Radiation Laboratory (Daresbury, UK) or at beam line X-33 of EMBL, c/o DESY (Hamburg, Germany). Both facilities were set up to detect small-angle (SAXS) and wide-angle (WAXS) X-Ray scattering intensities, respectively, so as to allow correlation between mesophase repeat spacings and packing arrangements of the acyl chains. X-ray scattering intensity profiles were normalized for the incident beam intensity measured using an incident beam ionization chamber and for the channel detector response calibrated with a static radioactive iron source. Calibration of the camera (1 or $2 \mathrm{~m}$ length) for $\mathrm{d}$-spacings ( $X$-axis) were obtained using rat tail collagen $(65 \mathrm{~nm})$ or silver behenate $(5.838 \mathrm{~nm})$ for SAXS and with $p$-bromobenzoic acid or high density polyethylene for WAXS. Data analysis was carried out with the OTOKO program kindly provided by M.H.J. Koch [20].

\section{Results}

\subsection{Influence of sphingomyelin on phospholipid substrate}

The effect of sphingomyelin on the structure and phase behavior of a phosphatidylethanolamine (PE)/phosphatidylserine (PS) (4:1) mixed dispersion has been examined by synchrotron X-ray diffraction methods in an attempt to explain the effect on membrane enzymes of sphingomyelin [21,22]. Fig. 1 shows that in the absence of sphingomyelin a dispersion of phosphatidylethanolamine: phosphatidylserine (80:20) is phase separated at $37^{\circ} \mathrm{C}$. This is characterized by Bragg reflections in the small-angle scattering region showing a strong reflection at $d_{\text {spacing }}=5.2$ $\mathrm{nm}\left(\mathrm{S}^{-1}=0.192\right)$ with a weaker second-order reflection at $2.6 \mathrm{~nm}\left(\mathrm{~S}^{-1}=0.385\right)$ and a group of reflections at $d_{\text {spacing }}=5.8,3.4$ and $2.9 \mathrm{~nm}\left(\mathrm{~S}^{-1}=\right.$ $0.172,0.294$ and 0.345).

The intense scattering centered at $5.2 \mathrm{~nm}$ is assigned to a lamellar phase which is comprised predominantly of PS. Previous studies indicate the preferred arrangement of PS is lamellar $[17,18]$. The short periodicity suggests that the phase is partially dehydrated. The group of other reflections are in a space grouping $1: 1 / \sqrt{ } 3: 1 / 2$ which is assigned to the inverted hexagonal-II structure expected for the PE component of the mixture. This assignment is consistent with the stability of the hexagonal phase at higher temperatures and the parallel decrease in intensity of all three reflections on cooling. The successive changes in structure of the mixed dispersion on cooling can be seen from the sequence of X-ray diffraction patterns in the small (Fig. 1a) and wide-angle (Fig. 1b) regions recorded over the temperature range $45^{\circ}$ to $0^{\circ} \mathrm{C}$. A gel phase first appears at approximately $33^{\circ} \mathrm{C}$ typified by a sharp wide-angle reflection at $0.42 \mathrm{~nm}$ indicating an ordered packing of the acyl chains. The transition is likely to be a lamellar liquid-crystal to lamellar gel phase transition of the phase-separated PS 


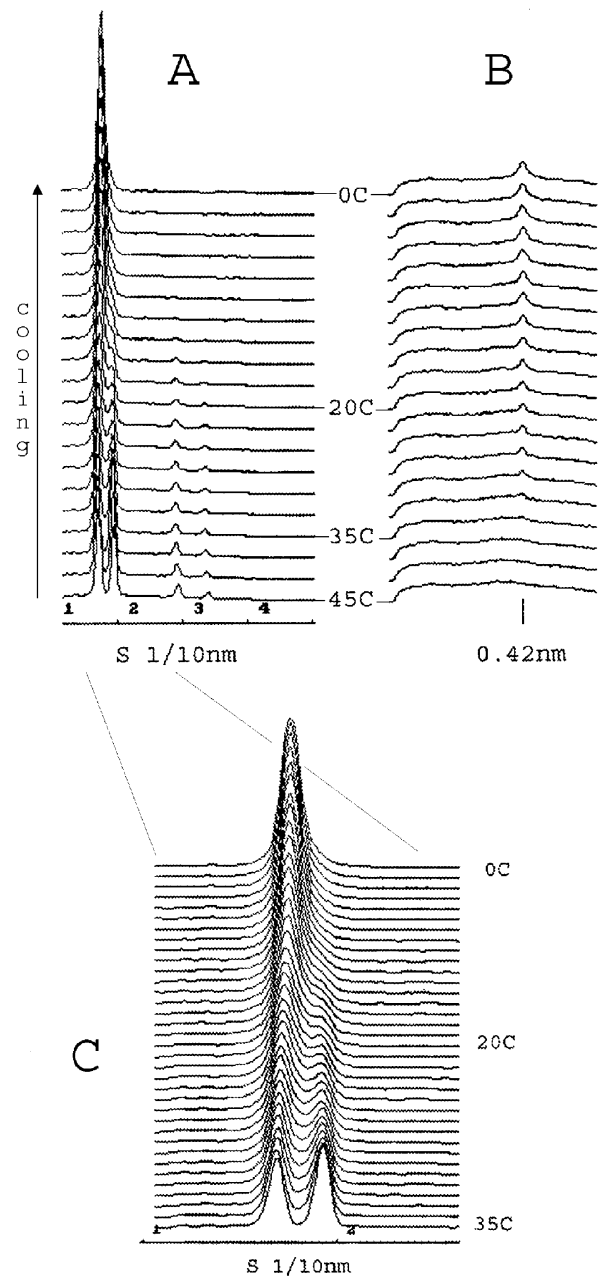

Fig. 1. Small-angle (a) and wide-angle (b) X-ray scattering intensity recorded from a mixed aqueous dispersion of phosphatidylethanolamine (PE): phosphatidylserine (PS) (4:1, $\mathrm{mol} / \mathrm{mol}$ ) hydrated (100\%: w/w) with $100 \mathrm{mM}$ Tris $-\mathrm{HCl}$ aqueous buffer, $\mathrm{pH} 8,10 \mathrm{mM}$ calcium, during a cooling scan from $45^{\circ}$ to $0^{\circ} \mathrm{C}$ at $2^{\circ} / \mathrm{min}$. (c) Detail of the small-angle X-ray scattering intensity in the temperature range of the $\mathrm{H}_{\mathrm{II}} /$ lamellar phase transition.

with a broad temperature range of approximately $27^{\circ} \mathrm{C}$ which reflects the heterogeneous acyl chain composition of the phospholipid. The absence of calcium in the buffer does not affect phase separation of the two phospholipids at temperatures above $35^{\circ} \mathrm{C}$ but decreases the fluid-to-gel phase mid-transition temperature to $24^{\circ} \mathrm{C}$ (data not shown). Cooling PE-PS co-dispersions below $30^{\circ} \mathrm{C}$ results in a decrease of the scattered intensity of peaks assigned to hexagonal-II phase which gradually transforms into a lamellar phase of periodicity $5.7 \mathrm{~nm}\left(\mathrm{~S}^{-1}=0.175\right)$ in a process that is completed at approximately $12^{\circ} \mathrm{C}$ (Fig. 1c). In (Fig. 1c) the first-order peak of the lamellar phase of PS centered at $5.2 \mathrm{~nm}$ is seen to merge within a new peak assigned to a mixed phase comprising gel $\mathrm{PE} / \mathrm{PS}$.

Inclusion of sphingomyelin (SM) in the mixture in proportions of $\mathrm{PE} / \mathrm{PS} / \mathrm{SM}(4: 1: 2)$ causes a significant change in the structure and phase behavior of the dispersion. This can be seen in a temperature scan shown in Fig. 2 from $20^{\circ}$ to $50^{\circ} \mathrm{C}$ followed by a subsequent cooling scan from $50^{\circ}$ to $20^{\circ} \mathrm{C}$. The initial structure at $20^{\circ} \mathrm{C}$ consists of two orders of a strong lamellar repeat with a $d$-spacing of $6.2 \mathrm{~nm}\left(\mathrm{~S}^{-1}=0.161\right)$ and two orders of a weaker lamellar phase with a $d$-spacing of 5.6 $\mathrm{nm}\left(\mathrm{S}^{-1}=0.178\right)$ appearing as a shoulder for a sample temperature-equilibrated at $20^{\circ} \mathrm{C}$ (lowest

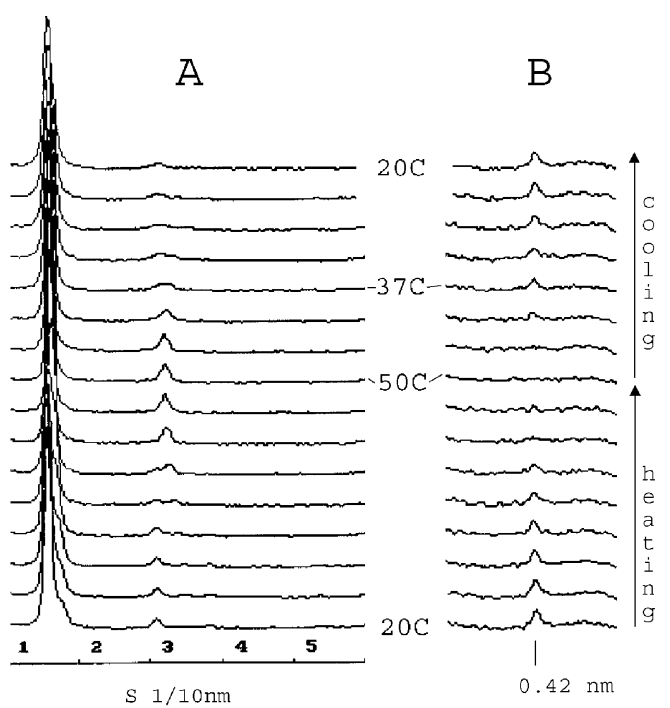

Fig. 2. Small-angle (a) and wide-angle (b) X-ray scattering intensity patterns recorded from a mixture of phosphatidylethanolamine: phosphatidylserine: sphingomyelin $(4: 1: 2, \mathrm{~mol} / \mathrm{mol})$ hydrated $(100 \%: \mathrm{w} / \mathrm{w})$ in $100 \mathrm{mM}$ Tris $-\mathrm{HCl}$ buffer, $\mathrm{pH} 8$, containing $10 \mathrm{mM}$ calcium. The temperature is scanned in the direction of the arrow from 20 to $50^{\circ} \mathrm{C}$ and back to $20^{\circ} \mathrm{C}$ at a scan rate of $1.5^{\circ} \mathrm{C} / \mathrm{min}$. 
SAXS profile in (Fig. 2a). After the temperature cycling the final recording at $20^{\circ} \mathrm{C}$ (highest SAXS profile in Fig. 2a shows a broadened reflection but no shoulder is resolved, an indication that a long equilibration is required for phase separation to take place. The persistence of a gel phase in the mixture at temperatures above approximately $40^{\circ} \mathrm{C}$, as evidenced by the sharp wide-angle reflection at $0.42 \mathrm{~nm}$ (Fig. 2b), is consistent with a gel separated lamellar SM coexisting with the lamellar fluid phase of diacylphospholipids. It is noteworthy that such a diffraction peak was not detected above $36^{\circ} \mathrm{C}$ in the binary mixture of $\mathrm{PE} / \mathrm{PS}$. This suggests that the long acyl chains associated with some molecular species of egg SM were responsible for formation of this separated gel phase at temperatures above approximately $40^{\circ} \mathrm{C}$. Because SM also prevents the phase separation of hexagonal-II phase of PE over the entire range of temperatures it is expected that other low melting point molecular species of SM with the shorter acyl chains may be intermixed within the fluid phospholipid phase.

The solubility of SM within the fluid phospholipids has been investigated as a function of temperature and equilibration time. The heterogeneity of the apparently broadened first order reflection (highest SAXS profile in Fig. 2a can be demonstrated after prolonged incubation at a temperature slightly below the fluid-to-gel transition temperature of SM. This can be seen from the results presented in Fig. 3. On initial cooling of the dispersion from $39^{\circ} \mathrm{C}$ to $33^{\circ} \mathrm{C}$ (lower panel of Fig. 3) a sharp lamellar reflection assigned to the gel phase of SM at $6.2 \mathrm{~nm}$ dominates the SAXS pattern and a weaker reflection appears as a shoulder centered at $5.6 \mathrm{~nm}\left(\mathrm{~S}^{-1}=0.178\right)$ due to the lamellar disordered liquid-crystal phase consisting predominantly of diacylglycerophospholipids. After $2 \mathrm{~h}$ equilibration at $33^{\circ} \mathrm{C}$ a change in the relative intensities of the two peaks was recorded which we interpret to reflect a slow partitioning of lower melting point molecular species of SM into fluid PE/PS. Therefore the phase-separation between viscous gel and fluid components of SM within the tertiary mixture PE:PS:SM is a time-dependant process at a tem-

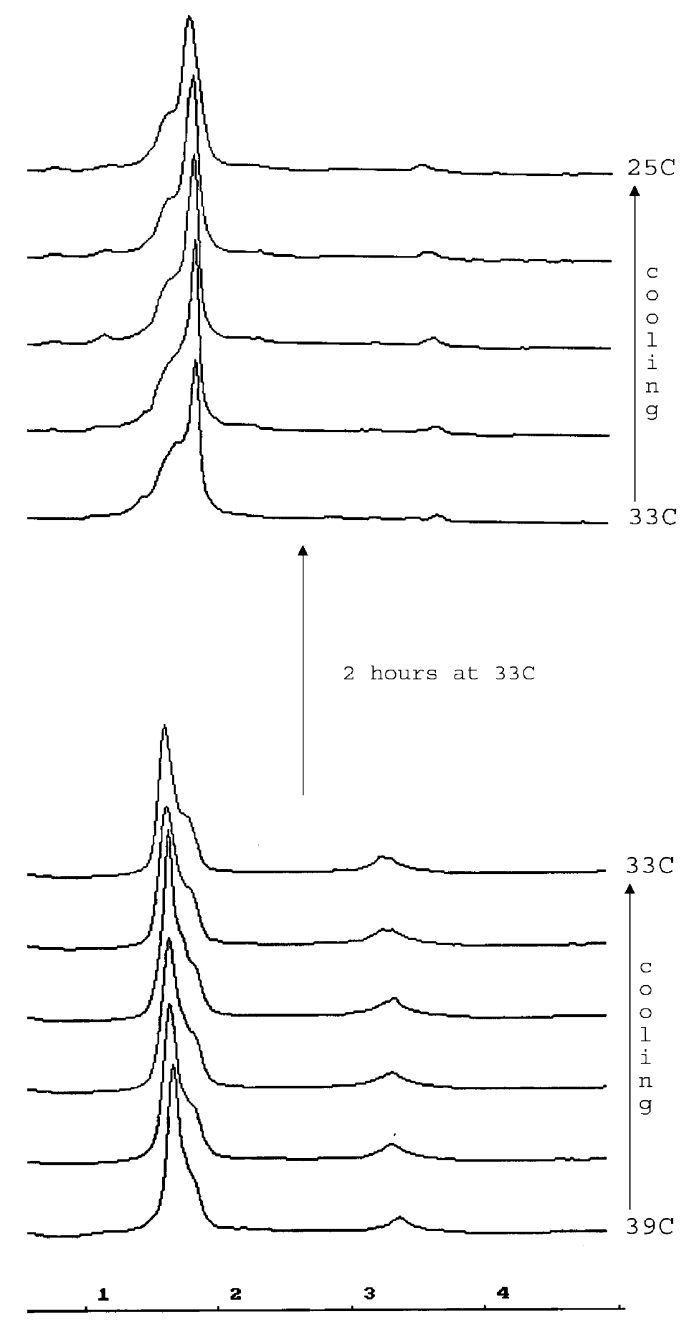

S $1 / 10 \mathrm{~nm}$

Fig. 3. Variation as a function of equilibration time of the small angle X-ray scattering of phosphatidylethanolamine: phosphatidylserine: sphingomyelin $(4: 1: 2, \mathrm{~mol} / \mathrm{mol})$ hydrated (100\%: w/w) in $100 \mathrm{mM}$ Tris-HCl buffer, $\mathrm{pH} 8$, containing 10 $\mathrm{mM}$ calcium. The temperature is initially scanned down in the direction of the arrow from 39 to $33^{\circ} \mathrm{C}$ and the scanning stopped at $33^{\circ} \mathrm{C}$. X-Ray exposure and cooling are resumed after $2 \mathrm{~h}$ during which the temperature of $33^{\circ} \mathrm{C}$ is kept constant.

perature close to physiological values. The phase separation is not observed if the temperature was scanned at a high rate of $2.5^{\circ} \mathrm{C} / \mathrm{min}$. This can be seen from Fig. 4 which shows that the first two 


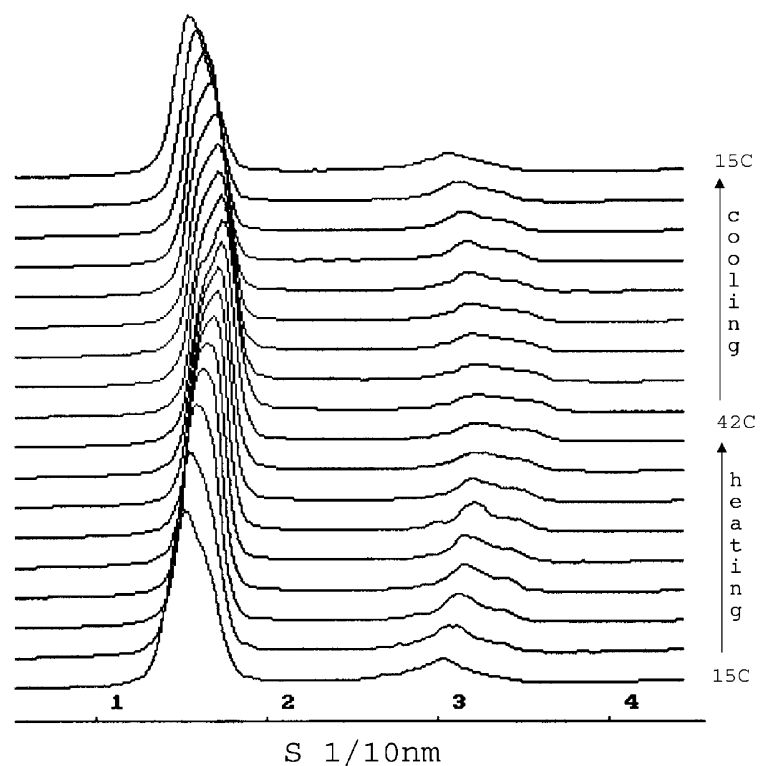

Fig. 4. Rapid temperature scanning of the small angle X-ray scattering of phosphatidylethanolamine: phosphatidylserine: sphingomyelin $(4: 1: 2, \mathrm{~mol} / \mathrm{mol})$ hydrated $(100 \%$ : w/w) in 100 $\mathrm{mM}$ Tris- $\mathrm{HCl}$ buffer, $\mathrm{pH} 8$, containing $10 \mathrm{mM}$ calcium. The temperature is scanned up and down between $15-40^{\circ} \mathrm{C}$ at the rate of $2.5^{\circ} \mathrm{C} / \mathrm{min}$.

orders of the lamellar repeat spacings are apparently distorted during the heating and cooling cycle but a close examination of the pattern reveals its heterogeneity.

To check whether SM was an essential factor for phase separation within the dispersion a mixture consisting of egg PE/PS/egg PC (4:1:2) was examined. The thermotropic properties of this dispersion are illustrated in Fig. 5 which shows the identical SAXS intensity profiles during heating from 30 to $45^{\circ} \mathrm{C}$. A single lamellar phase with a constant $d$-spacing of $6.1 \mathrm{~nm}\left(\mathrm{~S}^{-1}=0.164\right)$ exists over the entire temperature range investigated. WAXS data (not shown) indicates that the unsaturated acyl chain packing is disordered. The absence of any evidence of hexagonal-II phase suggest that, like sphingomyelin, phosphatidylcholine also possesses a tendency to impose a bilayer configuration on phosphatidylethanolamine. In addition the miscibility of all fluid compounds in the mixture produce a distinctive bilayer periodicity.

\subsection{Influence of sterols}

The presence of cholesterol in dispersions containing sphingomyelin facilitates phase separation. This is typified in a quaternary mixture (PE/PS/SM/CHOL) subjected to a heating scan $\left(2^{\circ} \mathrm{C} / \mathrm{min}\right.$ ) (Fig. 6a) followed by the immediate cooling scan (Fig. 6b). The first-order lamellar diffraction is split with an additional peak fully resolved below approximately $35^{\circ} \mathrm{C}$. The $d$-spacing of the latter peak is reduced on cooling whereas at temperatures of approximately $40^{\circ} \mathrm{C}$ the major diffraction appears only broadened. The additional diffraction peak has a $d$-spacing of $5.5 \mathrm{~nm}\left(\mathrm{~S}^{-1}=0.182\right)$ at $25^{\circ} \mathrm{C}$ and a weak secondorder reflection can be detected at $2.7 \mathrm{~nm}\left(\mathrm{~S}^{-1}=\right.$ 0.370 ). By contrast the $d$-spacing of the major lamellar phase increases progressively on cooling from a value of $6.03 \mathrm{~nm}\left(\mathrm{~S}^{-1}=0.166\right)$ at $37^{\circ} \mathrm{C}$ to $6.37 \mathrm{~nm}\left(\mathrm{~S}^{-1}=0.157\right)$ at $20^{\circ} \mathrm{C}$. The phase sequence is reversed with no observed hysteresis (Fig. 6a,b) which precludes the de-mixing on a macroscopic scale of one of the components in the dispersion. It is noteworthy that cholesterol prevents formation of any gel phase detectable in

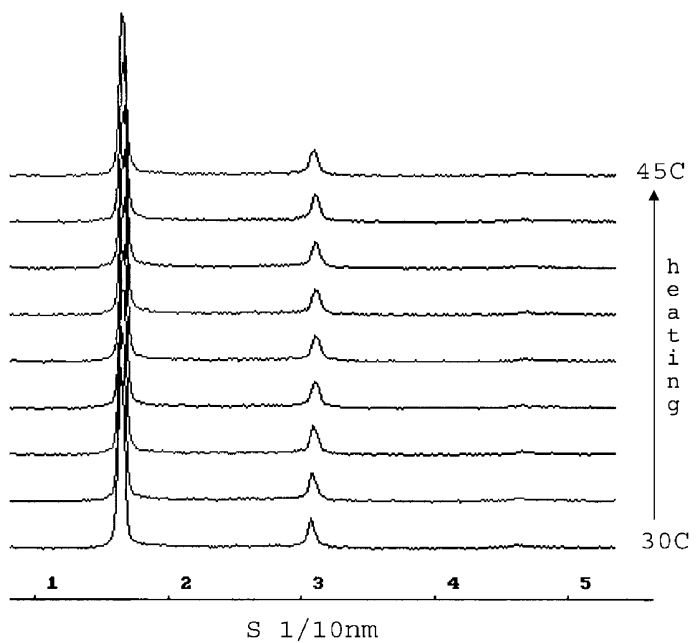

Fig. 5. SAXS intensity profiles of a mixed dispersion of phosphatidylethanolamine: phosphatidylserine: phosphatidylcholine $(4: 1: 2, \mathrm{~mol} / \mathrm{mol})$ hydrated $(100 \%$ : w/w) in $100 \mathrm{mM}$ Tris- $\mathrm{HCl}$ buffer $(\mathrm{pH} 8), 10 \mathrm{mM}$ calcium. The temperature scan from 30 to $45^{\circ} \mathrm{C}$ was at $2^{\circ} \mathrm{C} / \mathrm{min}$. 

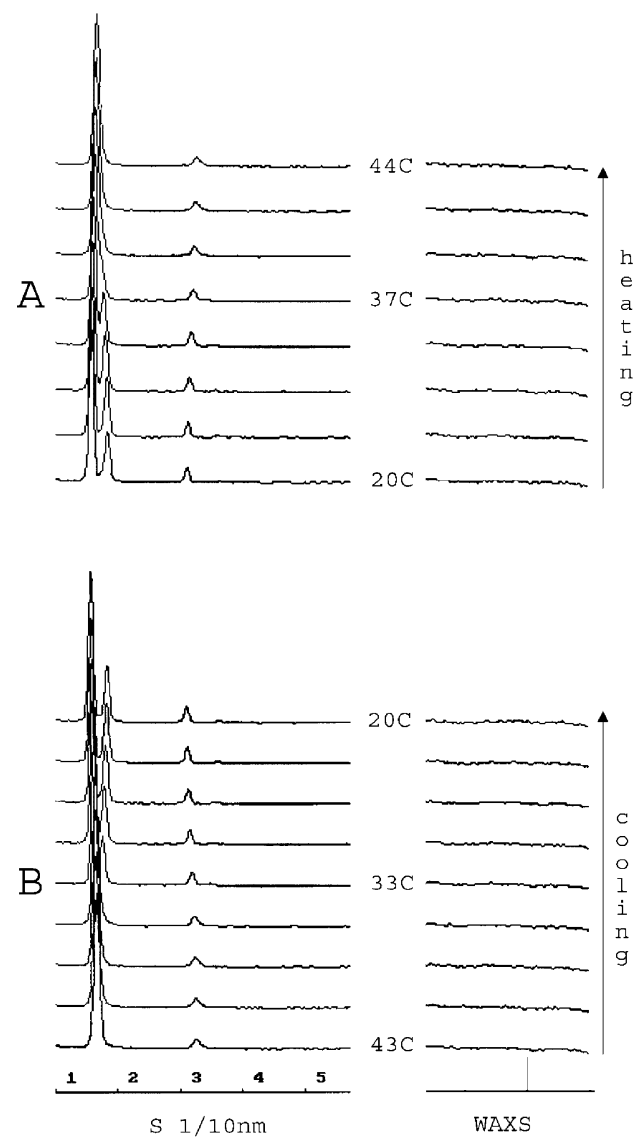

Fig. 6. SAXS and WAXS intensity profiles of a mixed dispersion of phosphatidylethanolamine: phosphatidylserine: sphingomyelin: cholesterol $(4: 1: 2: 2, \mathrm{~mol} / \mathrm{mol})$ hydrated $(100 \%$ : $\mathrm{w} / \mathrm{w})$ in $100 \mathrm{mM}$ Tris- $\mathrm{HCl}$ buffer $(\mathrm{pH} 8), 10 \mathrm{mM}$ calcium. The dispersion was heated (a) from $20^{\circ} \mathrm{C}$ to $44^{\circ} \mathrm{C}$ and subsequently cooled (b) to $20^{\circ} \mathrm{C}$ at $2^{\circ} / \mathrm{min}$. The tick bar of the WAXS $\mathrm{X}$-axis indicates $0.42 \mathrm{~nm}$.

the WAXS intensity profiles which are characterized by a diffuse scattering throughout the range of the temperature scans.

To determine whether interaction between PS and excess $\mathrm{Ca}^{2+}$ influences the phase separation within $\mathrm{PE} / \mathrm{PS} / \mathrm{SM} / \mathrm{CHOL}$ mixtures were prepared without added calcium (Fig. 7a). The SAXS intensity patterns recorded during heating and cooling scans (Fig. 7a) show that phase separation takes place also in the absence of calcium but the onset for the detection of the additional peak is detected on cooling at a lower temperature $\left(34^{\circ} \mathrm{C}\right)$.
The particular role of cholesterol structure to generate phase separation was clearly supported by a comparison with 7-dehydrocholesterol (7DHC) in (Fig. 7b). The SAXS profiles for an aqueous dispersion in which 7-DHC, the immediate precursor in the biosynthesis pathway, substitutes for cholesterol show that the lamellar re-
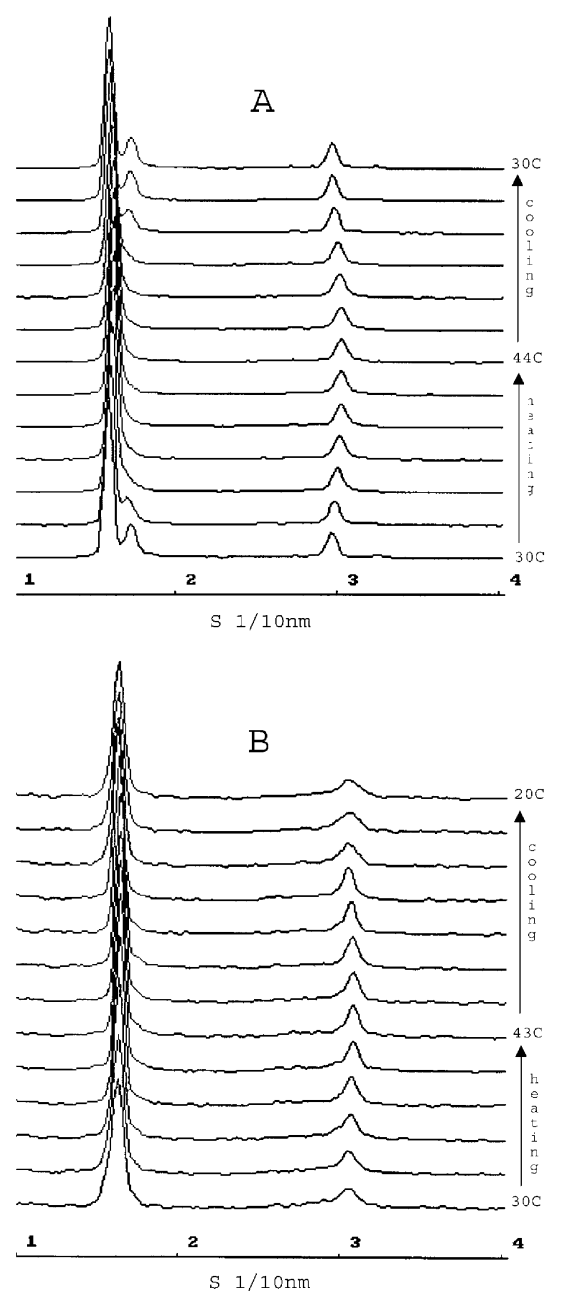

Fig. 7. SAXS intensity profiles of a mixed dispersion of (a) phosphatidylethanolamine: phosphatidylserine: sphingomyelin: cholesterol $(4: 1: 2: 2, \mathrm{~mol} / \mathrm{mol})$ and (b) phosphatidylethanolamine: phosphatidylserine: sphingomyelin: 7dehydrocholesterol $(4: 1: 2: 2, \mathrm{~mol} / \mathrm{mol})$ hydrated $(100 \%: \mathrm{w} / \mathrm{w})$ in $100 \mathrm{mM}$ Tris- $\mathrm{HCl}$ buffer, $\mathrm{pH} 8$ (no added calcium). The dispersions were heated from 30 to $44^{\circ} \mathrm{C}$ and subsequently cooled to 30 (a) or $20^{\circ} \mathrm{C} \mathrm{(b)} \mathrm{at} 2^{\circ} / \mathrm{min}$. 
peat spacing broadens at temperatures below $30^{\circ} \mathrm{C}$ but no evidence of an additional lamellar phase appears within the dispersion at temperatures down to $20^{\circ} \mathrm{C}$. As with cholesterol, the absence of WAXS reflections shows the non-ordered acyl chain arrangement in the temperature range investigated resulting of the intermixing of the sterol within the lipids (not shown).

\section{Discussion}

Hydrolysis of substrate dispersions comprised of PE/PS mixtures by the inflammatory secretory type II phospholipase $\mathrm{A}_{2}$ is inhibited in the presence of SM but not PC; full recovery of activity is achieved by the presence of cholesterol in amounts equimolar with SM [22]. Studies of enzyme activity have hitherto been regarded as a predictive index of the physical state of lipid substrate especially with respect to phase-separation believed to expose 'boundary structure defaults' which allow enzyme penetration and attack.

Choline-containing phospholipids (PC and SM) constitute most of the lipids of the outer leaflet in 'resting' plasma membranes [23-25] where they fulfil a structural role. The presence of sphingomyelin $[25,26]$ in the inner membrane leaflet which is enriched in amino-glycerophospholipids (PE/PS) together with cholesterol is believed to form the detergent-resistant microdomains, the so called 'liquid-ordered rafts', which interact with GPI-anchored proteins [6]. The present study of SM embedded in a fluid amino-glycerophospholipid matrix appear useful to model the inner leaflet of the plasma membrane since the previous studies of mixtures consisting of SM: PC are more representative of the stable lamellar external leaflet arrangement [10]. The partitioning of cholesterol is also of particular interest as it is known to be correlated with localization of sphingomyelin $[25,26]$ with which it forms the liquid-ordered $\left(L_{\mathrm{o}}\right)$ phase which coexists with the fluid matrix [27,28]. The limited solubility of cholesterol in the surrounding PE/PS $[12,14,15]$ and the segregation of gel SM from the fluid matrix could also participate in their co-localization.

An initial conclusion drawn from the present data is that SM phase-separates within the fluid phase of mixed aqueous dispersions of aminoglycerophospholipids. The formation of a gel phase at temperatures below $40^{\circ} \mathrm{C}$ or of a liquidordered $\left(L_{\mathrm{o}}\right)$ phase with cholesterol creates a phase separation within the mixed dispersion. Binary mixtures of $\mathrm{PE} / \mathrm{PS}$ in the absence of $\mathrm{SM}$ display a clear phase separation of two different arrangements at temperatures above the transition temperatures of both lipids; the inverted hexagonal $\mathrm{H}_{\text {II }}$ phase was assigned to polyunsaturated $\mathrm{PE}$ and the lamellar phase to PS $[17,18]$. This latter component has a short lamellar periodicity $(5.2 \mathrm{~nm})$ which allows a clear distinction between the two phases on the basis of SAXS intensity profiles. On cooling below $33^{\circ} \mathrm{C}$ a gel phase is formed which is characterized by a sharp wide-angle reflection at $0.42 \mathrm{~nm}$ resulting from the ordered hexagonal packing of the hydrocarbon chains. An assignment to a PS/Ca complex is confirmed because the gel-to-fluid transition temperature is widely influenced by the presence of calcium. The fluid-to-gel transition temperature of PS precedes by more than $13^{\circ} \mathrm{C}$ the disappearance of the hexagonal phase of PE below $20^{\circ} \mathrm{C}$. At lower temperatures a single lamellar phase of periodicity $5.8 \mathrm{~nm}$ is formed as presumably the two lipids mix in the resulting lamellar phase. The gradual mixing suggests that the PS which has the stronger tendency to form a lamellar phase slowly incorporates into PE on cooling thereby promoting an hexagonal to lamellar transition of the PE.

Addition of SM to PE/PS mixed dispersions also serves to shift the balance of the phase equilibrium towards the lamellar arrangement. However, the phase of phospholipids within the bilayer which is formed by the ternary mixture remains heterogeneous at physiological temperatures. Two lamellar phase periodicities are detected at temperatures below the gel-to-fluid transition of the SM after sufficient temperature equilibration. The incorporation of unsaturated PC into the PE/PS mixed dispersion, by contrast, eliminates any heterogeneity within the disper- 
sion. This infers that fluid PC in proportions of from 20 to $30 \%$ mole exerts a strong phase-preference which dominates the arrangement of the entire lipid mixture so that only a single lamellar phase ( $d$-spacing $6.1 \mathrm{~nm}$ ) is observed. The influence of SM on the binary mixture PE/PS appears to be similar but in a more stepped manner as a function of the temperature. Like PC, sphingomyelin reduces the propensity of polyunsaturated $\mathrm{PE}$ to organize into the phase separated inverted hexagonal structure shown by previous ${ }^{31} \mathrm{P}-\mathrm{NMR}$ studies [17,18]. Below the mean transition temperature, we assign the periodicity characterized by a $d$-spacing of $6.2-6.0 \mathrm{~nm}$ to a lamellar phase of sphingomyelin in the gel phase and the lamellar phase of $d$-spacing $5.6 \mathrm{~nm}$ to the fluid amino-phospholipids intermixed with the low melting point SM species. These molecular species display a similar capability to PC in preventing the separation of an inverted hexagonal phase of PE. The high melting point SM is likely to be comprised mainly of very long chain molecular species which explains the longer $d$-spacing and the existence of the gel state evident from the WAXS region at high temperatures.

It is noteworthy that cholesterol prevents the formation of any gel phase comprising high melting point SM species or PS/calcium because WAXS profiles of the mixtures are characterized by a diffuse scattering throughout the temperature scans. The association of the sterol with the alkyl/acyl chains of SM below $40^{\circ} \mathrm{C}$ forms the liquid-ordered lamellar phase $\left(L_{\mathrm{o}}\right)[3,7]$ assigned to the lamellae of periodicity $6.37 \mathrm{~nm}$ at $20^{\circ} \mathrm{C}$ which separates readily on cooling from the fluid glycerophospholipids (periodicity $5.6 \mathrm{~nm}$ ). The greater thickness of $L_{\mathrm{o}}$ compared with $L_{\alpha}$ may assist in separation of the phases. Because of its thickness $L_{\mathrm{o}}$ was claimed to participate in the re-location of long trans-membrane peptide sequences [29]. Cholesterol appears in the present investigation to facilitate the separation of SM containing phase as a function of the temperature scanning rate. Thus, within a mixture where two lamellar (gel/fluid) phases coexist the addition of cholesterol facilitates their separation possibly as the result of accelerated lateral diffusion. We have recently observed using ESR spectroscopy a motional regime for spin probes characterizing $L_{\mathrm{o}}$ in mixtures containing a sterol mixed to $\mathrm{PE} / \mathrm{PS} / \mathrm{SM}$ [30]. Cholesterol and $\Delta 7$-dehydrocholesterol gave a highly anisotropic component below $36^{\circ} \mathrm{C}$ but above this critical temperature only cholesterol could maintain a stable association with SM. This remarkable feature of the precursor $\Delta 7$-dehydrocholesterol is confirmed in the present study by the finding that no splitting of the long periodicity assigned to the liquidordered phase can be detected under experimental conditions where cholesterol associates with $\mathrm{SM}$. Whereas the cooling rate $\left(-2^{\circ} \mathrm{C} / \mathrm{min}\right)$ was sufficient for the cholesterol: SM association to emerge, the lower affinity for the 7DHC: SM association impaired the organization of a diffractive unit. We conclude that the interaction between the 2 rigid chemical structures, i.e. cholesterol and gel SM, is stringent in fastresponding biological membranes approximately physiological temperatures.

\section{Acknowledgements}

K. Koumanov has received during the completion of this study a grant from CNRS (UPRESA 7079) and from Université P. et M. Curie. B. Tenchov acknowledges the invaluable help of Dr Gert Rapp and Dr Michel Koch with the X-ray measurements and software programmes.

\section{References}

[1] S.J. Singer, G.L. Nicholson, The fluid mosaic model of the structure of cell membranes, Science 175 (1972) $720-731$.

[2] E.K. Fridrikson, P.A. Shipkova, E.D. Sheets, D. Holowka, B. Baird, F.W. McLafferty, Quantitative analysis of phospholipids in functionally important membrane domains from RBL-2H3 mast cells using tandem high-resolution mass spectroscopy, Biochemistry 38 (1999) 8056-8063.

[3] D.A. Brown, E. London, Functions of lipid rafts in biological membranes, Annu. Rev. Cell Dev. Biol. 14 (1998) 111-136.

[4] D.A Brown, E. London, Structure of detergent resistant membrane domains: Does phase separation occur in biological membranes? Biochem. Biophys. Res. Commun. 240 (1997) 1-7. 
[5] M.B. Sankaran, T.E. Thompson, Cholesterol-induced fluid-phase immiscibility in membranes, Proc. Natl. Acad. Sci. USA 88 (1991) 8686-8690.

[6] R.J. Schroeder, S.N. Ahmed, Y. Zhu, E. London, D.A. Brown, Cholesterol and sphingolipid enhance the Triton X-100-insolubility of GPI-anchored proteins by promoting the formation of detergent insoluble ordered membrane domains, J. Biol. Chem. 273 (1998) 1150-1157.

[7] S.N. Ahmed, D.A. Brown, E. London, On the origin of sphingomyelin-cholesterol rich detergent-insoluble domains in cells membranes: physiological concentrations of cholesterol and sphingolipid induce formation of a detergent-insoluble liquid-ordered lipid phase in models membranes, Biochemistry 36 (1997) 10944-10953.

[8] R. Schroeder, E. London, D. Brown, Interactions between saturated acyl chains confer detergent resistance on lipids and GPI-anchored proteins: GPIanchored proteins in liposomes and cells show similar behavior, Proc. Natl. Acad. Sci. USA 91 (1994) 12130-12134.

[9] X Xu, E. London, The effect of sterol structure on membrane lipid domains reveals how cholesterol can induce lipid domain formation, Biochemistry 39 (1994) 843-849.

[10] W.I. Calhoun, G.G. Shipley, Sphingomyelin-lecithin bilayers and their interaction with cholesterol, Biochemistry 18 (1979) $1717-1722$.

[11] P.R. Maulik, G Shipley, X-Ray diffraction and calorimetric study of N-lignoceryl sphingomyelin membranes, Biophys. J. 69 (1995) 1909-1916.

[12] J Huang, G.W. Feigenson, A microscopic interaction model of maximum solubility of cholesterol in lipid bilayers, Biophys. J. 76 (1999) 2142-2157.

[13] T.P.W. McMullen, R.N.A.H. Lewis, R.N. McElhaney, Calorimetric and spectroscopic studies of the effects of cholesterol on the thermotropic phase behavior and organization of a homologous series of linear saturated phosphatidylethanolamine bilayers, Biochim. Biophys. Acta. 1416 (1999) 119-134.

[14] J. Huang, J.T. Buboltz, G.W. Feigenson, Maximum solubility of cholesterol in phosphatidylcholine and phosphatidylethanolamine bilayers, Biochim. Biophys. Acta. 1417 (1999) 89-100.

[15] R.M. Epand, D. Bach, N. Borochov, E. Wachtel, Cholesterol crystalline polymorphism and the solubility of cholesterol in phosphatidylserine, Biophys. J. 78 (2000) 866-873.

[16] J.A.F. Op den Kamp, Lipid asymmetry in membranes., Annu. Rev. Biochem. 48 (1979) 47-71.

[17] C.P Tilcock, P.R Cullis, The polymorphic phase behaviour of mixed phosphatidylserine-phosphatidylethanolamine model systems as detected by ${ }^{31} \mathrm{P}-\mathrm{NMR}$, Biochim. Biophys. Acta. 641 (1981) 189-201.

[18] M.B Bally, C.P Tilcock, M.J. Hope, P.R. Cullis, Polymorphism of phosphatidylethanolamine-phosphati- dylserine model systems: influence of cholesterol and $\mathrm{Mg}^{2+}$ on $\mathrm{Ca}^{2+}$-triggered bilayer to hexagonal (HII) transitions, Can. J. Biochem. Cell Biol. 61 (1983) 346-352.

[19] H. Katsikas, C. Wolf, Blood sphingomyelins from two European countries, Biochim. Biophys. Acta. 1258 (1995) 95-100.

[20] C. Boulin, R. Kempf, A. Gabriel, M.H.J. Koch, S.M. McLaughlin, Data appraisal, evaluation and display for synchrotron radiation experiments: hardware and software, Nucl. Instrum. Methods A249 (1986) 399-409.

[21] K.S. Koumanov, G. Bereziat, C. Wolf, Modulation of human type II secretory phospholipase A2 by sphingomyelin and annexin VI, Biochem. J. 326 (1997) 227-233.

[22] K.S. Koumanov, P.J. Quinn, G. Bereziat, C. Wolf, Cholesterol relieves the inhibitory effect of sphingomyelin on type II secretory phospholipase A2, Biochem J. 336 (1998) 625-630.

[23] R.F.A. Zwaal, B. Roelofsen, P. Comfurius, L.L.M. van Deenen, Organization of phospholipids in human red cell membranes as detected by the action of various purified phospholipases, Biochim. Biophys. Acta. 406 (1975) 83-96.

[24] J.P.J. Boegheim, M. van Linde, J.A.F. Op den Kamp, B. Roelofsen, The sphingomyelin pools in the outer and inner layer of the human erythrocyte membrane are composed of different molecular species, Biochim. Biophys. Acta. 735 (1983) 438-442.

[25] J.P. Slotte, G. Hedstrom, S. Rannstrom, S. Ekman, Effects of sphingomyelin degradation on cell cholesterol oxidizability and steady-state distribution between the cell surface and the cell interior, Biochim. Biophys. Acta. 985 (1989) 90-96.

[26] D. Allan, Mapping the lipid distribution in the membranes of BHK cells, Mol. Membrane Biol. 13 (1996) 81-84.

[27] C. Reyes Mateo, A. Ulises Acuna, J.C. Brochon, Liquid-crystalline phases of cholesterol/lipid bilayers as revealed by the fluorescence of trans-parinaric acid, Biophys. J. 68 (1995) 978-987.

[28] M.B. Sakaram, T.E. Thompson, Modulation of phospholipid acyl chain order by cholesterol. A solid-state ${ }^{2} \mathrm{H}$ nuclear magnetic resonance study, Biochemistry 29 (1990) 10670-10675.

[29] J. Ren, S. Lew, Z. Wang, E. London, Transmembrane orientation of hydrophobic alpha-helices is regulated both by the relationship of helix length to bilayer thickness and by the cholesterol concentration, Biochemistry 36 (1997) 10213-10220.

[30] C. Wolf, C. Chachaty, Compared effects of cholesterol and 7-dehydrocholesterol on sphingomyelinglycerophospholipid bilayers studied by ESR, Biophys. Chem. 84 (2000) 269-279. 